\title{
Missed Diagnosis and Treatment Dilemma: Large Patent Ductus Arteriosus Combined with Anomalous Origin of the Left Coronary Artery From the Pulmonary Artery
}

\author{
Ayca Ata Korkmaz, ${ }^{1,}{ }^{*}$ Hakan Erkan, ${ }^{2}$ Aysegul Karadeniz, ${ }^{2}$ and Cihan Orem ${ }^{3}$ \\ ${ }^{1}$ Department of Radiology, Kanuni Research and Education Hospital, Trabzon, Turkey \\ ${ }^{2}$ Department of Cardiology, Ahi Evren Cardiovascular and Thoracic Surgery Training and Research Hospital, Trabzon, Turkey \\ ${ }^{3}$ Department of Cardiology, Faculty of Medicine, Karadeniz Technical University, Trabzon, Turkey \\ "Corresponding author: Ayca Ata Korkmaz, Department of Radiology, Kanuni Research and Education Hospital, Trabzon, Turkey. Tel: +90-4623415656, Fax: +90-3447777, E-mail: \\ dr.h.aycaatakorkmaz@gmail.com
}

Received 2015 June 19; Revised 2015 August 16; Accepted 2015 August 30.

\begin{abstract}
An anomaly of the left coronary artery, originating from the pulmonary trunk, is called anomalous origin of the left coronary artery from the pulmonary artery (ALCAPA) syndrome. This syndrome is an infrequent congenital anomaly, mostly seen in children. Usually, because of the anomaly, myocardial perfusion failure occurs. This anomaly presents even less frequently in adults. The symptoms of ALCAPA develop in the early ages. Congestive heart failure is an important result of ALCAPA, as well as mitral insufficiency and left ventricular (LV) dysfunction. If there is a patent ductus arteriosus (PDA) or ventricular septal defect (VSD), high pulmonary artery pressures decreases and the coronary anomaly and perfusion defect can be hidden. Here we present a case of a large PDA combined with ALCAPA, as seen in one of the oldest patients ever reported. Recognizing PDA in patients with ALCAPA is very important, since closure of the PDA may cause catastrophic conditions. ALCAPA combined with PDA is very rare and has been reported mostly in infants. To the best of our knowledge, there is no such case diagnosed in adulthood.
\end{abstract}

Keywords: Coronary Artery Anomalies, Patent Ductus Arteriosus, Computerized Tomography

\section{Introduction}

The anomalous origin of the left coronary artery from the pulmonary artery (ALCAPA) is an infrequent congenital anomaly that develops in 1 in 300,000 live births and accounts for less than $0.5 \%$ of all congenital heart anomalies $(1,2)$. ALCAPA patients can exhibit signs of congestive heart failure associated with left ventricular dysfunction, which can occur in early infancy. The detrimental effect of ALCAPA on left ventricular (LV) function may remain asymptomatic, if there is a patent ductus arteriosus (PDA) reducing pulmonary arterial pressure that enables abnormal coronary artery perfusion. Recognizing PDA in patients with ALCAPA is very important, since closure of the PDA may cause catastrophic conditions.

A few cases of ALCAPA with PDA have been reported in infants. On the other hand, there has been no adult patient diagnosed with the combination of ALCAPA and PDA. In this case report, we present an adult patient, 22 years of age, who had a large PDA combined with ALCAPA and is one of the oldest patients ever reported.

\section{Case Presentation}

A 22-year-old woman with complaints of dyspnea and palpitations for a long period of time presented to the cardiology clinic. After evaluation, the patient was considered to have primary pulmonary hypertension and was referred for cardiac catheterization and pulmonary vasoreactivity testing. The patient's blood pressure measurement on admission was $140 / 78 \mathrm{~mm} \mathrm{Hg}$ and heart rate was $94 \mathrm{bpm}$. Physical examination findings and routine biochemical laboratory tests revealed no gross abnormality. Posterioranterior (PA) chest X-ray examination showed a slight enlargement in the cardiac anatomy with normal pulmonary vessels. Transthoracic echocardiography demonstrated normal left ventricular systolic function, moderate dilatation of right ventricle and a severely dilated pulmonary artery with systolic pressure of $140 \mathrm{mmHg}$. Color Doppler echocardiography showed moderate tricuspid valvular regurgitation and trivial mitral regurgitation. For the evaluation of the primary pulmonary hypertension, left and right ventricular catheterization was planned. The patient was taken to the catheterization laboratory. The right femoral vein was cannulated with a $6 \mathrm{~F}$ sheath. During the attempts with a multipurpose catheter to achieve the main pulmonary artery, the catheter ended up in another loca- 
tion. A contrast agent was given to check the position of the catheter. This demonstrated that the catheter was in the subclavian artery (Figure 1A). Then, the left femoral artery was punctured to perform aortography. Aortography confirmed the diagnosis of PDA(Figure 1B). The PDA was a large size with a high contrast enhancement level. Routine right cardiac catheterization procedures, including vasoreactivity testing (negative), were performed. The patient was considered to have a diagnosis of severe pulmonary artery hypertension due to the large PDA.

Thoracic computed tomography (CT) with and without a contrast agent is routinely ordered for patients with $\mathrm{PPH}$, in order to exclude possible pulmonary pathologies before cardiac catheterization. In thorax CT without a contrast agent, there was a suspicion of coronary abnormality and that the left coronary artery (LCA) was originating from the pulmonary artery. CT angiography demonstrated an anomalous origin of the LCA from the pulmonary artery. Also, a dilated main pulmonary truncus was detected. Multi-slice computed tomography confirmed the previously mentioned coronary abnormality and enlarged PDA (Figures 2 - 5).

\section{Discussion}

ALCAPA, also known as bland-white-garland syndrome, is an infrequent congenital cardiac anomaly. Normal coronary arteries are developed from: 1 ) endothelial sprouts localized in the walls of great arteries at the level of the sigmoidal values; 2 ) the right and left subepicardial vascular network; and 3) the intramyocardial sinusoids. Most of the ectopic coronary arteries are due to the alterations in the connection between these three embryonic elements. The deviation of one of the subepicardial vascular networks in the wrong way (in the direction of the pulmonary artery or the opposite valsalva sinus) will cause the development of endothelial sprouts, which will connect the abnormal connections and the anomalous origin of the coronary arteries (3).

PDA is classified into two subtypes. These subtypes are the infantile subtype and the adult subtype. In the infantile subtype, the symptoms occur at ages younger than 12 months, and in the adult subtype the symptoms occur at ages older than 12 months (4). Patients with this abnormality may present with subtle clinical complaints. Identifying these abnormalities is crucial in terms of medical and surgical management of patients. Poorly oxygenated blood from the coronary artery, with insufficient collateral circulation from the right coronary artery, and also the left coronary artery from the pulmonary artery, steals blood from the pulmonary circulation revealed by low coronary perfusion pressure due to the creation of myocardial ischemia symptoms.

Prenatally, there is no adverse effect that develops from ALCAPA because of the unique intrauterine fetal circulation; in the fetal circulation system, oxygen levels are equal between the main pulmonary artery and the aorta. Shortly after birth, a decrease in pressure, resistance and the oxygen content of the pulmonary artery leads to hypoperfusion of the myocardium by desaturated blood under low pressure. Without treatment, this syndrome is $90 \%$ fatal in the first year of life. Death is secondary to widespread myocardial ischemia, infarction, and at the end, developing of heart failure. Approximately $10 \%$ of cases are diagnosed in adulthood due to exertional chest pain and dyspnea caused by inadequate myocardial perfusion. In extremely rare cases, ALCAPA without symptoms is accidentally detected on cardiac screening in adults. But syncope, angina pectoris, dyspnea or cardiac arrest occurrence is more usual than an asymptomatic course with ALCAPA (5-7). After the development of a significant posttricuspid shunt, PDA prevents the fall in the pulmonary artery pressure, and PDA also provides oxygenated blood to the left coronary artery system, thus preventing the dramatic changes seen in a child with associated ALCAPA.

Conventional angiography is an invasive and limited diagnostic imaging modality for coronary artery anomalies. The sensitivity of conventional angiography is limited for the detection of a coronary artery anomaly because of its projection capability. CT coronary angiography is a beneficial non-invasive imaging method for demonstrating these types of coronary artery anomalies (8). However, multi-slice computed tomography (MSCT) coronary angiography is an excellent three-dimensional (3D) imaging method. Using this imaging modality, we were able to demonstrate the coronary vessel anatomy with high quality. In addition, another beneficial imaging modality for detecting ALCAPA is two-dimensional Doppler echocardiography. This diagnostic method is considered the best diagnostic tool for detecting ALCAPA (4).

Coronary artery anomalies originate from the left or right coronary artery, and both may be isolated or may be associated with different types of conotruncal and heart abnormalities (9-12). CT angiography is also a valuable tool for detecting other kinds of congenital cardiac abnormalities such as ventricular septal defect (VSD), atrial septal defect (ASD), PDA, tetralogy of Fallot, pulmonary atresia, Ebstein anomaly, transposition of the great arteries and tricuspid atresia. Ultrasonography is the best prenatal diagnostic imaging tool for intrauterine evaluation of the fetus. Also, congenital structural cardiac anomalies are easily detected by ultrasonography. However, coronary artery evaluation is not possible with ultrasonography. 

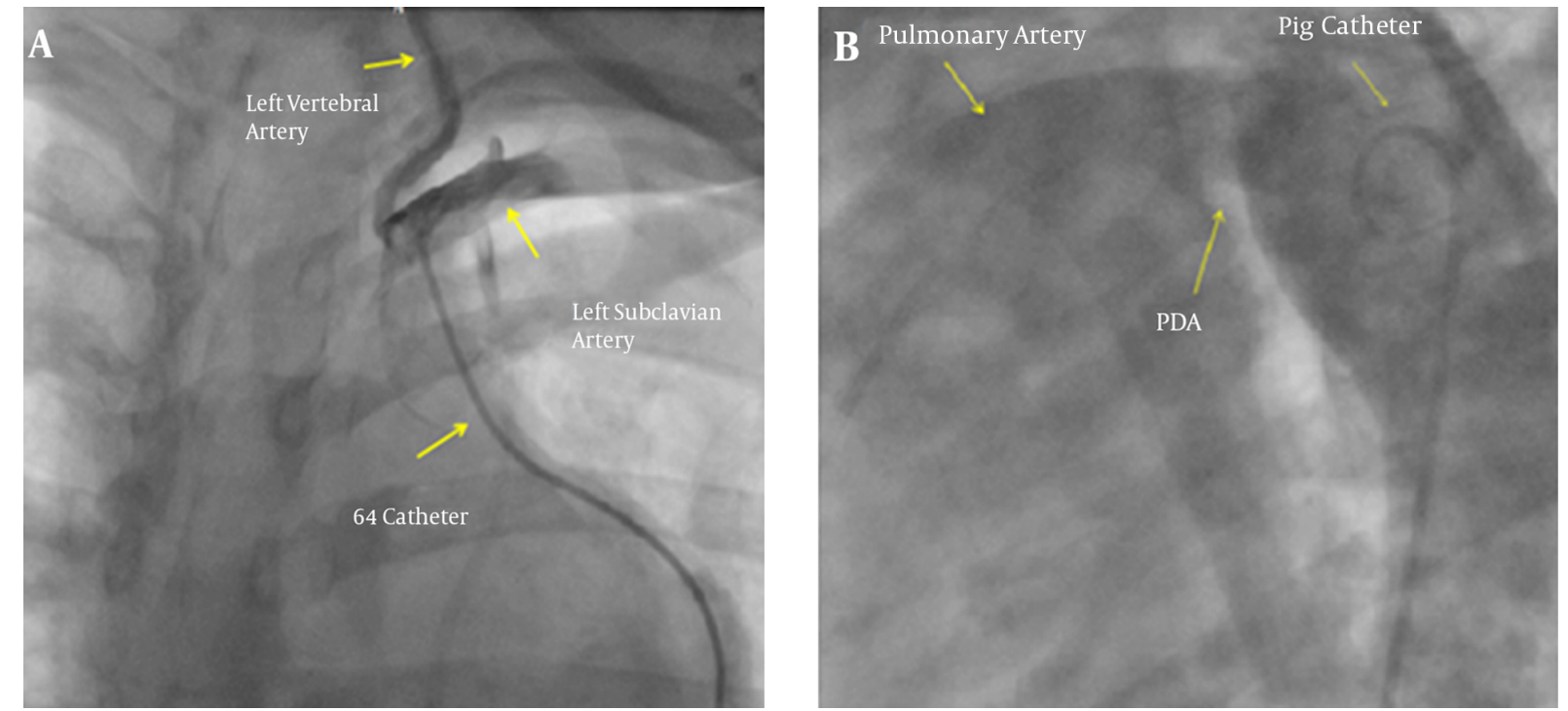

Figure 1. A 22-year-old woman with long term dyspnea and palpitations in whom primary pulmonary hypertension was detected. A, After right femoral vein catheterization with a $6 \mathrm{~F}$ sheath, the contrast agent demonstrated the left subclavian artery and we suspected PDA; B, aortography confirmed the diagnosis of PDA (PDA, patent ductus arteriosus).
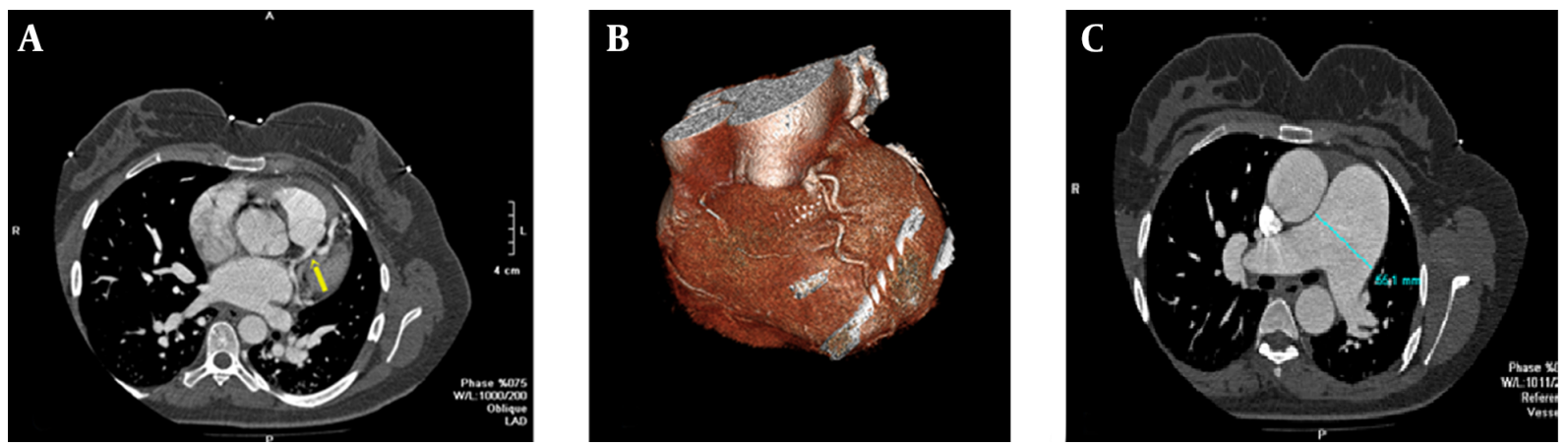

Figure 2. A, CT angiography shows the patient with an anomalous origin of the LCA from the pulmonary artery, also known as ALCAPA; B, CT angiography, three-dimensional reconstruction view of the ALCAPA; C, CT angiography shows dilated pulmonary truncus secondary to primary pulmonary hypertension, Abbreviations: LCA, left coronary artery; ALCAPA, anomalous origin of the left coronary artery from the pulmonary artery.

The treatment of choice for ALCAPA syndrome is surgical repair. Since early repair is associated with improvement in ventricular function in neonates, early repair is vital in the first months of life. Conservative medical treatment in asymptomatic older patients is rarely a reasonable alternative. However, asymptomatic adults with only limited infarction and moderate myocardial ischemia have a favorable survival rate without surgical repair. Generally, however, even if no left to right shunt symptoms are presented surgical correction is suggested for asymptomatic patients with ALCAPA. The American College of Cardiology and the American Heart Association guidelines suggest that surgical repair in adult patients is necessary to provide sufficient myocardial blood supply (13).

Treatment of our patient was not so straightforward. The patient had severe symptomatic pulmonary hypertension that warranted using an endothelin receptor antagonist, such as bosentan. We were not sure whether lowering the pulmonary artery pressure, at least on a small scale, would have a detrimental effect on left coronary pressure, resulting in left ventricular dysfunction. Since the patient had severe pulmonary hypertension, corrective surgery on coronary arteries carried a higher risk.

We think that this is the first case of adult type ALCAPA with PDA. Clinicians should be aware of this rare syndrome that may require a different approach in terms of design- 


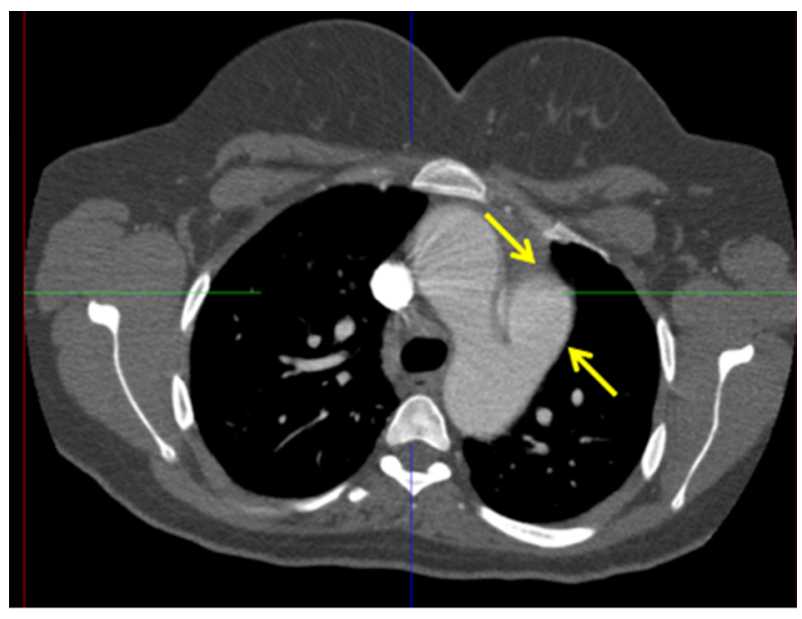

Figure 3. Contrast-enhanced axial CT image, patent ductus arteriosus depicting the persistent communication between the pulmonary artery and descending aorta (arrows).

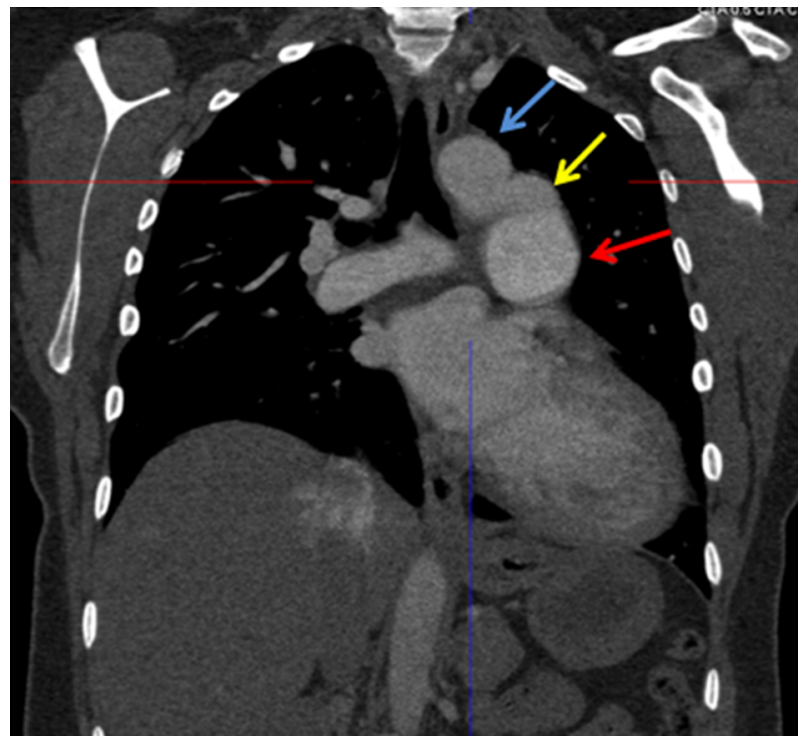

Figure 4. Contrast-enhanced coronal CT image, patent ductus arteriosus depicting the persistent communication between the pulmonary artery and descending aorta (yellow arrow), aorta (blue arrow), pulmonary artery (red arrow).

ing and treatment modalities. Also, patients with possible diagnosis of primary pulmonary hypertension should be evaluated in terms of very rare congenital abnormalities and undergo routine left cardiac catheterization, as well as coronary artery angiography, which should be performed in order not to miss rare vascular abnormalities.

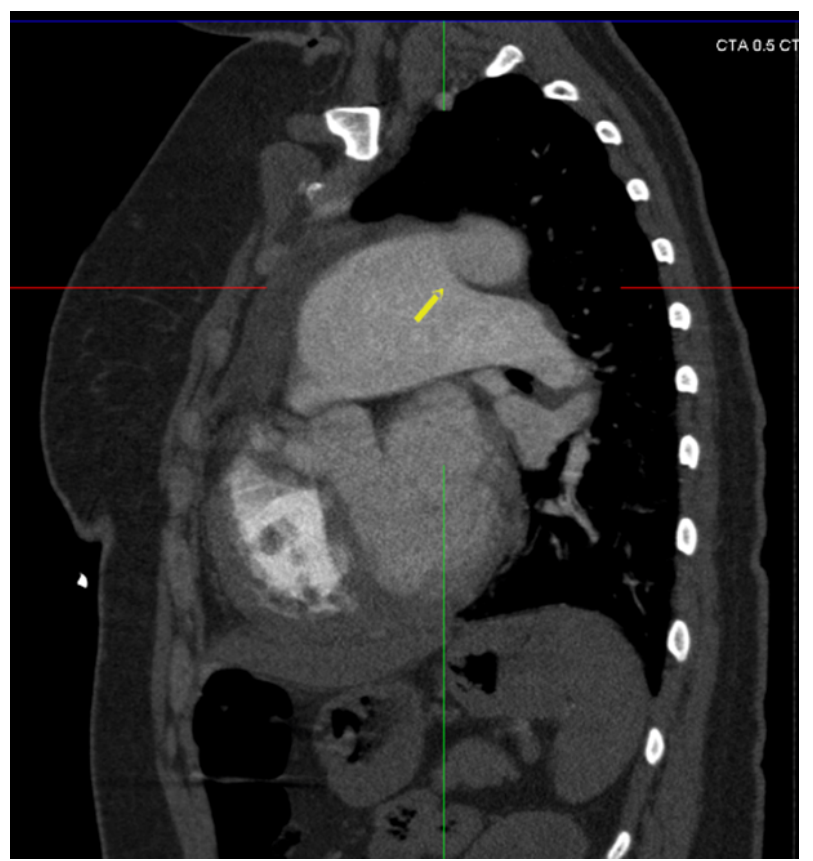

Figure 5. Contrast-enhanced sagittal CT image, patent ductus arteriosus depicting the persistent communication between the pulmonary artery and descending aorta (arrow).

\section{Footnote}

Authors' Contribution: Hakan Erkan performed cardiac catheterization procedures. Cihan Orem performed routine physical examinations. Ayca Ata Korkmaz and Aysegul Karadeniz assessed multislice cardiac computed tomography examinations. Ayca Ata Korkmaz wrote the manuscript. Hakan Erkan and Cihan Orem provided literature. And Aysegul Karadeniz reviewed the manuscripts in terms of grammetical errors.

\section{References}

1. Uruski P, Lipski D, Trojnarska O, Juszkat R, Tykarski A. ALCAPA syndrome in a 56-year-old woman with dyspnoea on exertion. Kardiol Pol. 2014;72(11):1165. [PubMed: 25671230].

2. Gribaa R, Slim M, Ben Salem H, Neffati E, Boughzela E. Anomalous origin of the left coronary artery from the pulmonary artery presenting as dilated cardiomyopathy: a case report. J Med Case Rep. 2014;8:170. doi: 10.1186/1752-1947-8-170. [PubMed: 24885797].

3. Rangel-Abundis A, Munoz-Castellanos L, Marin G, Chavez Perez E, Badui E. [Morphofunctional correlation in congenital anomalies of the coronary arteries. I. Coronary artery fistulas]. Arch Inst Cardiol Mex. 1994;64(2):161-74. [PubMed: 8074587].

4. Xiao Y, Jin M, Han L, Ding W, Zheng J, Sun C, et al. Two congenital coronary abnormalities affecting heart function: anomalous origin of the left coronary artery from the pulmonary artery and congenital left main coronary artery atresia. Chin Med J (Engl). 2014;127(21):3724-31. [PubMed: 25382327]. 
5. Orem C, Kiris A, Korkmaz L, Ozturk S, Kahraman N, Kosucu P, et al. Adult-type anomalous origin of the left coronary artery from the main pulmonary artery: one case report. Echocardiography. 2009;26(10):1232-5. doi: 10.1111/j.1540-8175.2009.00977.x. [PubMed: 19765067].

6. Frescura C, Basso C, Thiene G, Corrado D, Pennelli T, Angelini A, et al. Anomalous origin of coronary arteries and risk of sudden death: a study based on an autopsy population of congenital heart disease. Hum Pathol. 1998;29(7):689-95. [PubMed: 9670825].

7. Taylor AJ, Rogan KM, Virmani R. Sudden cardiac death associated with isolated congenital coronary artery anomalies. J Am Coll Cardiol. 1992;20(3):640-7. [PubMed: 1512344].

8. Ten Kate GJ, Weustink AC, de Feyter PJ. Coronary artery anomalies detected by MSCT-coronary angiography in the adult. Neth Heart J. 2008;16(11):369-75. [PubMed: 19065275].

9. Grünenfelder J, Zünd G, Vogt PR, Turina MI. Aortopulmonary window with anomalous origin of the right coronary artery. Ann Thorac Surg. 1999;67(1):233-5. doi: 10.1016/s0003-4975(98)01145-X.

10. McMahon CJ, DiBardino DJ, Undar A, Fraser CD Jr. Anomalous origin of left coronary artery from the right pulmonary artery in association with type III aortopulmonary window and interrupted aortic arch. Ann Thorac Surg. 2002;74(3):919-21. [PubMed:12238867].

11. Izumoto H, Ishihara K, Fujii Y, Oyama K, Kawazoe K. AP window and anomalous origin of right coronary artery from the window. Ann Thorac Surg. 1999;68(2):557-9. [PubMed:10475429].

12. Morell VO, Feccia M, Cullen S, Elliott MJ. Anomalous coronary artery with tetralogy of Fallot and aortopulmonary window. Ann Thorac Surg. 1998;66(4):1403-5. [PubMed: 9800843].

13. Warnes CA, Williams RG, Bashore TM, Child JS, Connolly HM, Dearani JA, et al. ACC/AHA 2008 guidelines for the management of adults with congenital heart disease: a report of the American College of Cardiology/American Heart Association Task Force on Practice Guidelines (Writing Committee to Develop Guidelines on the Management of Adults With Congenital Heart Disease). Developed in Collaboration With the American Society of Echocardiography, Heart Rhythm Society, International Society for Adult Congenital Heart Disease, Society for Cardiovascular Angiography and Interventions, and Society of Thoracic Surgeons. J Am Coll Cardiol. 2008;52(23):e143-263. doi: 10.1016/j.jacc.2008.10.001. [PubMed:19038677]. 